\title{
Upper limits for stereoselective photodissociation of free amino acids in the vacuum ultraviolet region and at the $\mathrm{C} 1 s$ edge
}

\author{
Georg Prümper,* Jens Viefhaus, Slobodan Cvejanović, Daniel Rolles, Oliver Geßner, ${ }^{\dagger}$ Toralf Lischke, Rainer Hentges, \\ Christian Wienberg, Willy Mahler, and Uwe Becker \\ Fritz-Haber-Institute der Max-Planck-Gesellschaft, 14195 Berlin, Germany \\ Burkhard Langer \\ Max-Born-Institut für Nichtlineare Optik und Kurzzeitspektroskopie, 12489 Berlin, Germany
}

Tommaso Prosperi, Nicola Zema, and Stefano Turchini

Istituto di Struttura della Materia CNR, Via Fosso del Cavaliere 100, 00133 Roma, Italy

Birgitt Zada

Max-Planck-Institut für Mikrostrukturphysik, Halle, Germany

Fred Senf

Berliner Elektronenspeicherring-Gesellschaft für Synchrotronstrahlung mbH (BESSY), 12489 Berlin, Germany

(Received 27 January 2004; published 25 June 2004)

\begin{abstract}
We measured the total and partial ion yields of the two chiral amino acids alanine and serine in the gas phase both in the vacuum ultraviolet region and at the $\mathrm{C}(1 s)$ edge using circularly polarized light. We did not detect any circular dichroism asymmetry larger than $1 \times 10^{-3}$. A similar measurement of fixed-in-space amino acids yielded an upper limit of $1 \times 10^{-2}$ for the stereoselective effect of circularly polarized light. The results obtained are relevant for quantitative models of stereoselective photodecomposition of amino acids that try to explain the homochirality of life.
\end{abstract}

DOI: 10.1103/PhysRevA.69.062717

PACS number(s): 33.80.Gj, 33.55.Ad, 33.15.Bh, 33.15.Ta

\section{INTRODUCTION}

Although more than 150 years have past since its discovery [1], the origins and implications of amino acid and sugar homochirality remain a frequently debated scientific topic [2,3]. Except for the electroweak interaction, all known forces do not violate the parity conservation. Especially the electromagnetic forces that determine chemistry and molecular structure give no preference to the $\mathrm{L}$ or the $\mathrm{D}$ form. This leads to the well-known consequence that chiral molecules always occur as racemic mixtures when they are created from achiral compounds in an achiral environment. It is still an unsolved question how - or if - chiral biology could have evolved from purely racemic substances. The L excess of amino acids found in the Murchison meteorite [4-6] looks like the missing link between a purely racemic scenario on the prebiotic earth and the complete homochirality of life. The analysis of the amino acids found in this meteorite yields an L excess of more than $10 \%$. All amino acids isolated from the cores of other carbonaceous meteorites are found invariably to be racemic.

The optical rotation of chiral molecules is connected with a difference in the absorption coefficients for circularly po-

\footnotetext{
*Present address: Institute of Multidisciplinary Research for Advanced Materials, Tohoku University, Sendai 980-8577, Japan.

${ }^{\dagger}$ Present address: Steacie Institute for Molecular Sciences, National Research Council Canada, Ottawa, Canada.
}

larized light of different helicity. If the absorption of the photon leads to a dissociation of the molecule, the illumination with circularly polarized light leads to a stereoselective decomposition of the molecules in a racemic sample and thus to an $\mathrm{L}$ or D excess in the remaining sample depending on the helicity and the wavelength of the light [7]. The circular dichroism of a homochiral sample is a measure of the selectivity of the circularly polarized light and thus its ability to optically enrich the sample in one enantiomer. Corresponding experiments in the optical region demonstrated that circularly polarized light can lead to a slight excess of the $\mathrm{L}$ or D form in a sample if only a narrow spectral region is used instead of white light. Norden reported on a $0.1 \%$ L enrichment of a alanine sample using a photon energy of $5.4-6.2 \mathrm{eV}$ [2]. The CD asymmetry of L-alanine is $0.1 \%$ at this photon energy. Camphor has a significantly higher value for the $\mathrm{CD}$ asymmetry of $4 \%$ at $3.3-4.3 \mathrm{eV}$. Balavoine and co-workers archived a enantiomeric enrichment of $20 \%$ by decomposing more than $99 \%$ of the original substance [8].

Several sources of naturally occurring polarized light are known to exist [3]. However, despite all evidence for the existence of stereoselective photodissociation, a very important inconsistency remains: the known values of the circular dichroism in the absorption are far to small to explain the findings in the Murchison meteorite. Independent of the open questions about the origin of life, this discovery needs an explanation based on hard experimental evidence and a model explaining the excess quantitatively. Balavoine and co-workers [8] pointed out that even a relatively small CD of $1 \%$ can lead to an $\mathrm{L}$ excess of $4 \%$ if $99 \%$ of the original 
substance is destroyed by the incident light. However, to explain an L excess of $10 \%$ with a CD asymmetry of $1 \%$, only a fraction of $4.5 \times 10^{-5}$ of the original mass could remain. Especially taking into account the limited degree of circular polarization of naturally occurring light sources, we therefore conclude that a $\mathrm{CD}$ of at least several percent is required to explain the $10 \%$ excess by photodissociation. In this context, we would like to point out that measurements show larger CD effects if the experimental setup defines a handed system and only a subset of all absorption events is considered. Angle resolved photoelectron experiments, for example, yield CD asymmetries of several percent for randomly oriented homochiral molecules [9,10], and theoretical predictions yield asymmetry values as high as 60\% [11]. Such effects vanish, however, if the observation is extended to all emission angles of the electrons and cannot explain an $\mathrm{L}$ excess in the remaining sample without further assumptions.

The availability of circularly polarized synchrotron radiation and the high accuracies obtainable by ion spectroscopy with these sources allow a precise measurement of the CD asymmetries in a wide range of photon energies. Due to the broad interest in high accuracy CD measurements, a number of dedicated devices has been set up at various synchrotron light sources. We performed ion yield measurements of free alanine $\left(\mathrm{C}_{3} \mathrm{H}_{7} \mathrm{NO}_{2}\right)$ and serine $\left(\mathrm{C}_{3} \mathrm{H}_{7} \mathrm{NO}_{3}\right)$ molecules in the vuv range and at the $\mathrm{C}(1 s)$ edge. Of course, ion spectroscopy cannot be used below the ionization threshold of $8 \mathrm{eV}$ [12]; therefore we could not compare our data to absorption measurements in the optical region.

\section{THE EXPERIMENTAL METHOD}

The amino acids L,D-alanine and L, D-serine were purchased from Sigma (purity of 99\%). Homochiral samples of L- and D-alanine and L- and D-serine were evaporated inside a vacuum chamber by careful heating to $140{ }^{\circ} \mathrm{C}$. This temperature is sufficiently lower than the decomposition temperature of more than $200{ }^{\circ} \mathrm{C}$. The problem of thermal sample decomposition is discussed by Holterkamp and coworkers [13], direct evidence for the presence of the amino acid molecules in the gas phase is given in [14]: chemical ionization experiments with source temperatures up to $520 \mathrm{~K}$ showed one single peak corresponding to an amino acid molecular ion $(M+\mathrm{H})^{+}$. Furthermore valence photoelectron spectra of gas phase alanine measured by Powis and coworkers [12] show clear evidence for intact alaninemolecules using an evaporation temperature of $268{ }^{\circ} \mathrm{C}$. As the samples contain a large amount of water, sample preparation involves drying at $80^{\circ} \mathrm{C}$ in the vacuum for $24 \mathrm{~h}$ prior to the measurement. The amino acid molecules are evaporated from a stainless steel container through a $1 \mathrm{~mm}$ diameter and $3 \mathrm{~mm}$ length opening. The beam intersects the ionizing synchrotron radiation after approximately $20 \mathrm{~mm}$ between two electrodes of the ion time-of-flight spectrometer. The mass spectra of the ionic fragments measured at the photon energy $22.5 \mathrm{eV}$ were consistent with the reference spectra from the NIST database [15] measured at an ionization energy of $70 \mathrm{eV}$. The fragment masses 28 and $44 \mathrm{amu}$

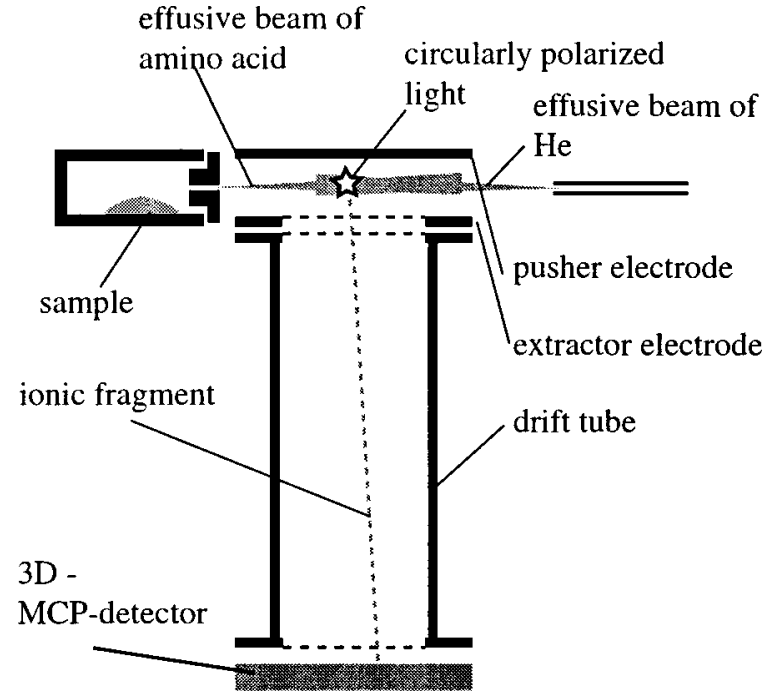

FIG. 1. The ion spectrometer is a three element Wiley-McLarentype time-of-flight spectrometer. A pulsed electric field is applied to the pusher. $+200 \mathrm{~V}$, the extractor electrode is grounded. The gap between the pusher and extractor is $8 \mathrm{~mm}$. A rectangular, $8 \mu \mathrm{s} \mathrm{du}-$ ration, $20 \mathrm{KHz}$ repetition rate pulse generator with a rise time of better than $20 \mathrm{~ns}$ was used. The detector consists of a segmented anode.

dominate the spectrum. The minor contributions include the masses 74 and 15. Assuming simple CC-bond breaking, the masses 15 and 74 amu belong to a separation of the $\mathrm{CH}_{3}$ group, the masses 44 and 45 belong to a separation of the $\mathrm{COOH}$ group, and the masses 15, 29, and 45 belong to the breaking of both CC-bonds. Using this scheme, the dominating mass 44 is assigned to the fragments $\mathrm{C}_{2} \mathrm{NH}_{6}$ or $\mathrm{CO}_{2}$. This indicates that the sample did not decompose due to the thermal evaporation even though the signal from the parent ion $(89 \mathrm{amu})$ is very weak due to the easily cleaved CCbonds.

The ionizing radiation was provided by third generation undulator beamlines (CIPO at ELETTRA, Trieste, Italy [16], and UE56/2 at BESSY II, Berlin, Germany [17]). Both beamlines do not only provide circularly polarized light with a high degree of circular polarization $\left|S_{3}\right|>0.8$ but allow a fast switching of the light helicity. The helicity can be inverted every $5 \mathrm{~s}$. This is a crucial feature in order to perform $\mathrm{CD}$ asymmetry measurements with an accuracy of better than $10^{-3}$, because this way, a slow change in the target density does not influence significantly the ratio of the count rates for $\sigma^{+}$and $\sigma^{-}$light. A pulsed field ion time-of-flight spectrometer of the Wiley-McLaren type [18] was used to determine the masses of the fragment ions (see Fig. 1). The spectrometer is equipped with a position sensitive anode which makes it possible to measure the momentum of all of the ionic fragments simultaneously [19]. Please refer to $[20,21]$ for further details.

A more common time-of-flight technique uses static extraction fields and an electron detector as a start signal and an ion detector as a stop signal for the time-of-flight measurement. Such a device could not be used because the electron detection usually depends on the emission angle of the electron. The large $\mathrm{CD}$ effects in the angular distribution of pho- 
toelectrons would introduce additional systematic errors. Worst of all, these systematic errors cannot be distinguished from the true CD signal by changing the enantiomer.

The quantity of interest is the relative change of the count rates $I_{L \text {-amino acid }}^{\sigma^{+}}$and $I_{L \text {-amino acid }}^{\sigma^{-}}$when the helicity of the light is changed and the light intensity is kept constant:

$$
(\mathrm{CD} \text { asymmetry })=\frac{I_{L \text {-amino acid }}^{\sigma^{+}}-I_{L \text {-amino acid }}^{\sigma^{-}}}{I_{L \text {-amino acid }}^{\sigma^{+}}+I_{L \text {-amino acid }}^{\sigma^{-}}} .
$$

This quantity is equal to the stereoselectivity of circularly polarized light:

$$
(\text { stereoselectivity })=\frac{\sigma_{L \text {-amino acid }}^{+}-\sigma_{D \text {-amino acid }}^{+}}{\sigma_{L \text {-amino acid }}^{+}+\sigma_{D \text {-amino acid }}^{+}} .
$$

At both beamlines used, a slight change of the intensity of some percent occurs, when the helicity of the light is switched. Therefore, the ion yield of a rare gas, e.g., helium, was used to monitor the light intensity during the measurement. A rare gas atom does not show a CD since it does not have a chiral structure. We normalized the count rates to the rare gas signal:

$$
\text { (normalized CD asymmetry) }=\frac{\frac{I_{L \text {-amino acid }}^{\sigma^{+}}}{I_{\text {rare gas }}^{\sigma^{+}}}-\frac{I_{L \text {-amino acid }}^{\sigma^{-}}}{I_{L \text {-amino acid }}^{\sigma^{-}}}+\frac{I_{L \text {-amino acid }}^{\sigma^{-}}}{I_{\text {rare gas }}^{\sigma^{+}}}}{I_{\text {rare gas }}^{\sigma^{-}}} .
$$

In this quantity, the change in the light intensity is eliminated. Depending on whether $I_{L \text {-amino acid }}^{\sigma^{+}}$refers to all ionic fragments or just one particular mass, we refer to it as either the "total CD asymmetry" or the "mass resolved CD asymmetry."

In addition to changes in the light intensity, systematic errors can occur due to changes of position of the light beam, photon energy changes, and due to scattered light of low energy. We took great care to avoid such systematic errors by monitoring the light spot position with a luminous screen, interchanging the role of the upstream and downstream undulator at the UE56/2 beamline, and the use of a low energy cutoff filter as well as by comparing the results found for Land D-amino acids which must show opposite sign in the CD asymmetries. As a results of these measures, we were able to reduce the systematic error of the the $\mathrm{CD}$ measurement to less than $10^{-3}$. Our accuracy is determined by the statistical error. We accumulated typically some $10^{6}$ ion counts in the amino acids and the rare gas signal.

\section{UPPER LIMITS FOR THE CD OF RANDOMLY ORIENTED CHIRAL MOLECULES IN THE VUV}

We measured the CD asymmetry of L- and D-alanine and $\mathrm{L}$ - and D-serine at various photon energies in the vuv region in the energy range from 10 to $30 \mathrm{eV}$. The results are

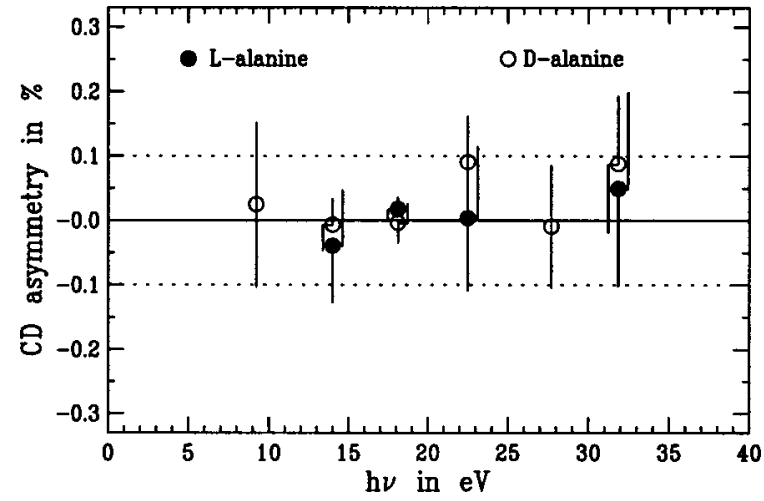

FIG. 2. The CD results for the total ion yield of randomly oriented chiral molecules in the vuv region.

shown in Fig. 2. Our data clearly show that a CD measurement with a precision of $1 \times 10^{-3}$ is possible in the gas phase using our experimental method. We found no evidence for $\mathrm{CD}$ in this energy range and can only give an upper limit of $1 \times 10^{-3}$ for the $\mathrm{CD}$ asymmetry. A similar result was found recently for 2-amino-1-butanol at a photon energy of $21 \mathrm{eV}$ by Mackie and co-workers [22] putting an upper limit of $10^{-2}$ for the CD asymmetry. This is consistent with the extrapolation of the optical measurements to higher photon energies [23].

One corresponding mass spectrum is shown in Fig. 3. The occurring masses can be assigned to the braking of the CCbonds. The mass resolved $\mathrm{CD}$ is expected to be more pronounced because different fragment masses belong to different fragmentation channels that may show CD of different strength or even different sign.

However, even in the mass resolved ion yield, we did not observe a significant $\mathrm{CD}$ and can only give an upper limit of $5 \times 10^{-3}$ for the fragments with 28 and 44 amu in alanine.

\section{UPPER LIMITS FOR THE CD OF RANDOMLY ORIENTED CHIRAL MOLECULES NEAR THE C(1s) EDGE}

At higher photon energies near the $\mathrm{C}(1 s)$ edge, the absorption cross section and the photon flux of the beamline is

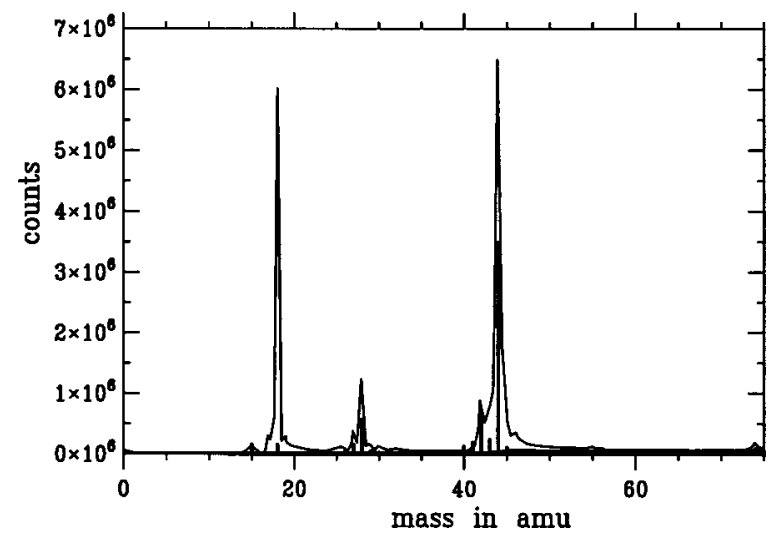

FIG. 3. The mass spectrum of L-alanine at $h \nu=18.1 \mathrm{eV}$ (curve). The lines correspond to the mass spectrum from the NIST database [15]. With the exception of a strong contribution at $18 \mathrm{amu}\left(\mathrm{H}_{2} \mathrm{O}\right)$, our data is consistent with Ref. [15]. 


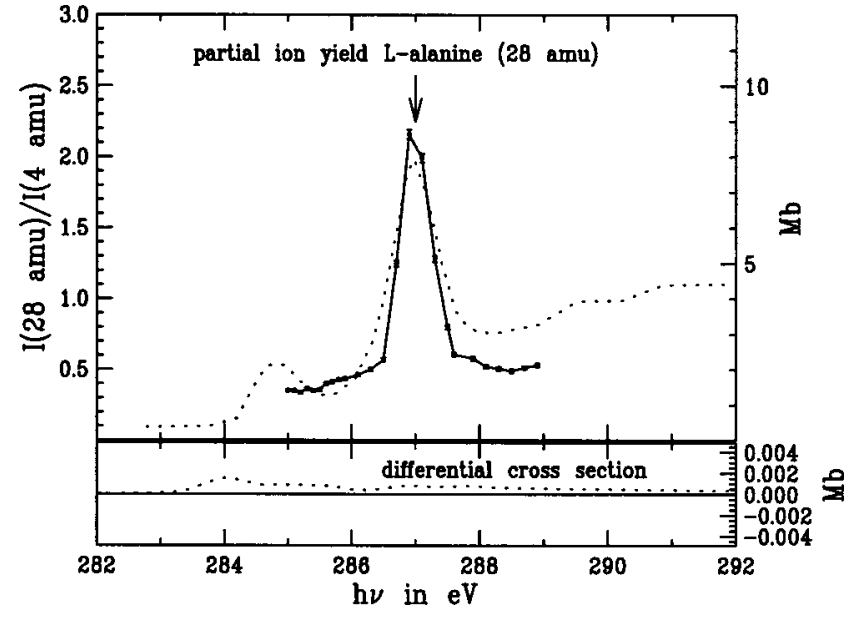

FIG. 4. Partial ion yield at the $\mathrm{C}(1 s)$ edge of L-alanine. The solid line is the measured partial ion yield signal, the dotted line is a theoretical calculation [24]. The left scale refers to the measured ion yields, while the right scale $(\mathrm{Mb})$ refers to the theoretical data. The lower plot shows the difference in the cross sections for light of opposite helicities. The photon energy resolution was better than $100 \mathrm{meV}$ full width at half maximum (FWHM) during this scan. The photon energies of the theoretical data were shifted by $-4.25 \mathrm{eV}$ to match the experimental data. The arrow indicates the energy of the CD measurement.

much smaller compared to the low photon energies. Therefore the time-consuming $\mathrm{CD}$ measurement that needs $10^{6}$ counts in the ion signal was only performed at a single photon energy for alanine and serine. In order to choose the most promising photon energy, we first measured the partial ion yield as a function of the photon energy with limited statistics. The helium ion yield was used to normalize the amino acid ion signals to the photon flux. Such a normalization method eliminates all features that originate from the various carbon containing surface contaminations of the optical elements of the beamline. The results are shown in Figs. 4 and 5.

The total ion yield of alanine shows a featureless broad peak without substructure for the three chemically different carbon atoms, while the serine data clearly shows the three edges. Unlike in alanine, the various fragments also behave very differently (not shown here). The featureless behavior of alanine, which we reproduced several times and with different samples, seems puzzling. For comparison, we measured the total and partial ion yield of the chemically similar alaninol, yielding features that can be assigned to the chemically different carbon atoms. These features are much less pronounced than in serine.

Due to the high photon energies near the $\mathrm{C}(1 s)$ edge, breaking of the molecules is not limited to the weak bonds anymore and the corresponding mass spectra are much richer than in the vuv region (compare Figs. 3, 6, and 7). The CD measurement was performed at the photon energies 287 and $306 \mathrm{eV}$ for alanine and serine, respectively. The positions are indicated with arrows in Figs. 4 and 5. In the case of serine, we chose this particular photon energy because the calculated CD values of Yang and co-workers [24] were maximal $6.5 \mathrm{eV}$ above the dominating peak. In the case of alanine we

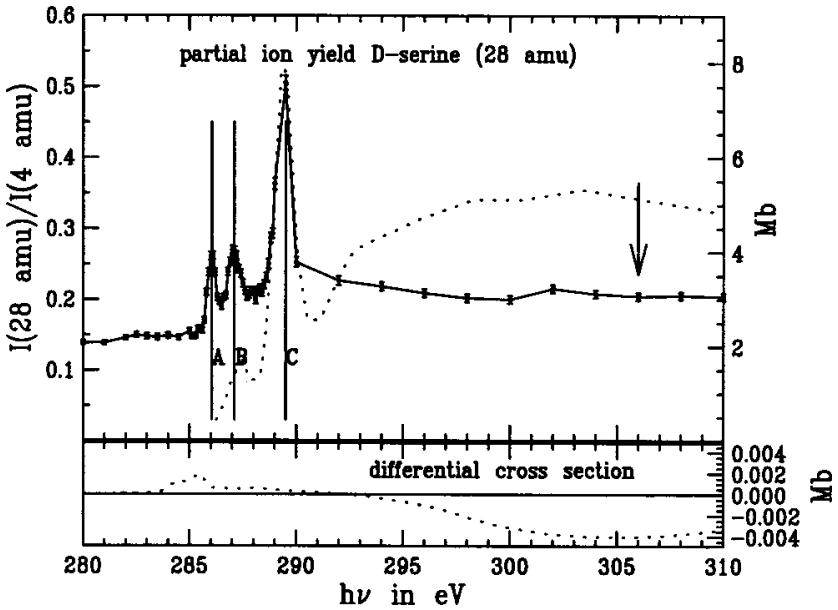

FIG. 5. Partial ion yield of D-serine at the $\mathrm{C}(1 s)$ edge. The solid line is the measured partial ion yield signal, the dotted line is theory [24]. The left scale refers to the measured ion yields, while the right scale $(\mathrm{Mb})$ refers to the theoretical data. The photon energy resolution was better than $100 \mathrm{meV}$ FWHM during this scan. Theory values have been shifted $-5.60 \mathrm{eV}$ in photon energy to match the experimental data. The arrow indicates the energy of the CD measurement. The chemical shifts of the different $\mathrm{C} 1 s$ edges are clearly resolved and contribute differently to the different fragment masses (not shown here).

choose the photon energy of the local maximum of the cross section.

The high degree of circular polarization $s_{3}>0.8$ of the two beamlines $[16,17]$ used for this experiment was checked for many different photon energies in various circular dichroism experiments. This value refers to the broad energy range from 20 to $500 \mathrm{eV}$. The only exception is the carbon edge where the light intensity of the beamline is reduced by absorption of carbon-containing contaminations of various optical elements. In contrast to common belief, recent measurement at the BESSY UE56/2 beamline showed that the polarization properties of the light are changed significantly by these contaminations. Circular polarization is partially transferred into linear polarization. This effect is significant in the photon energy region between 275 and $300 \mathrm{eV}$ where a linear polarization of more than 0.1 was found. Therefore we assume that the value of $s_{3}>0.8$ is correct for $306 \mathrm{eV}$. At

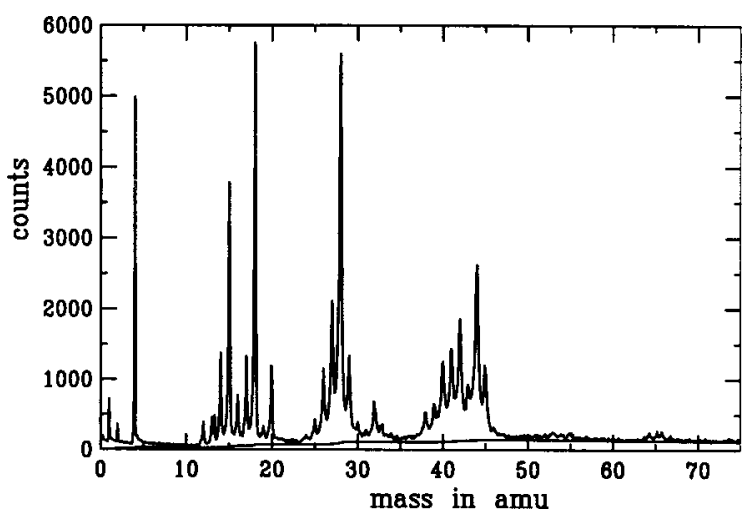

FIG. 6. The mass spectrum of L-alanine at $h \nu=287.1 \mathrm{eV}$. 


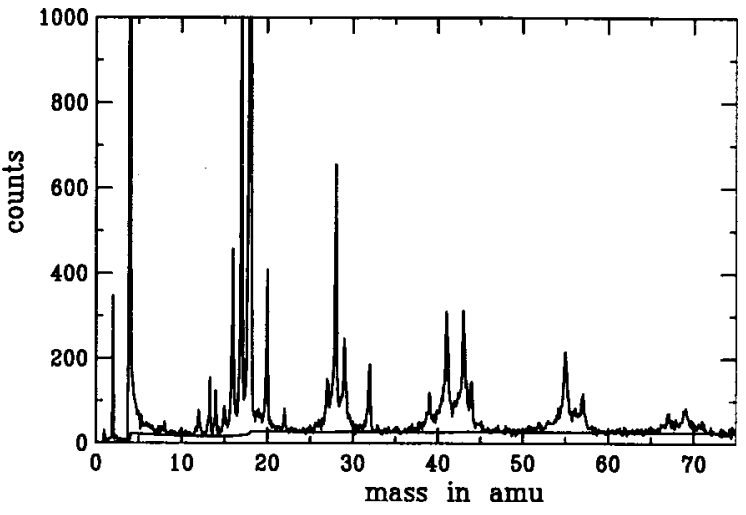

FIG. 7. The mass spectrum of D-serine at $h \nu=306.0 \mathrm{eV}$. This spectrum contains strong contributions of $\mathrm{H}_{2} \mathrm{O}$ coming from the oven, as the sample drying procedure was too short in this case.

$287 \mathrm{eV}$ a linear polarization of 0.4 was found, which corresponds to a reduction of $s_{3}$ from 0.80 to 0.69 assuming that the contaminations only transfer circular to linear polarization. We wish to point out that the CD measurement is only sensitive to the value $s_{3}$. Possible changes in the degree of linear polarization do not affect the measurement. Moreover, the $\mathrm{CD}$ measurement does not rely on the assumption that the absolute value of $s_{3}$ is exactly the same for the two helicities of the light. The size of the CD is simply proportional to the average value of $s_{3}$ and does not depend critically on the polarization properties of the light.

At both photon energies used, our data did not show any evidence for $\mathrm{CD}$ greater than $1 \times 10^{-3}$ in the total ion yield. For the mass resolved $\mathrm{CD}$ asymmetry, we found an upper limit of $5 \times 10^{-3}$ for all mayor masses in Figs. 6 and 7 .

\section{UPPER LIMITS FOR THE CD OF PARTIALLY ORIENTED CHIRAL MOLECULES}

The small CD of randomly oriented chiral molecules is a result of the interference of different photon-molecule interaction operators. Usually, it originates from the interference between the electric and magnetic dipole transition moments, but the electric dipole-electric quadrupole interference can sometimes be of the same order [25]. The smallness of these contributions is the reason for the small CD effects calculated. One way to increase the size of the $\mathrm{CD}$ effects is to increase the relative size of the higher multipole contributions by using light of shorter wavelength. The general trend of increasing importance of higher multipole contributions with photon energy is compensated by the fact that unlike the valence orbitals the core orbitals are almost spherical and thus basically achiral. The other approach to produce large $\mathrm{CD}$ asymmetries is to look for situations were $\mathrm{CD}$ effects are dipole allowed.

When only the electric dipole term is considered, the electric field is treated as a homogenous rotating field without a three-dimensional cork-screw structure. Such a field only defines a rotation direction in the plane perpendicular to the propagation direction of the light. Obviously, randomly oriented chiral molecules cannot distinguish between the two different rotation directions in a plane because the contribu-

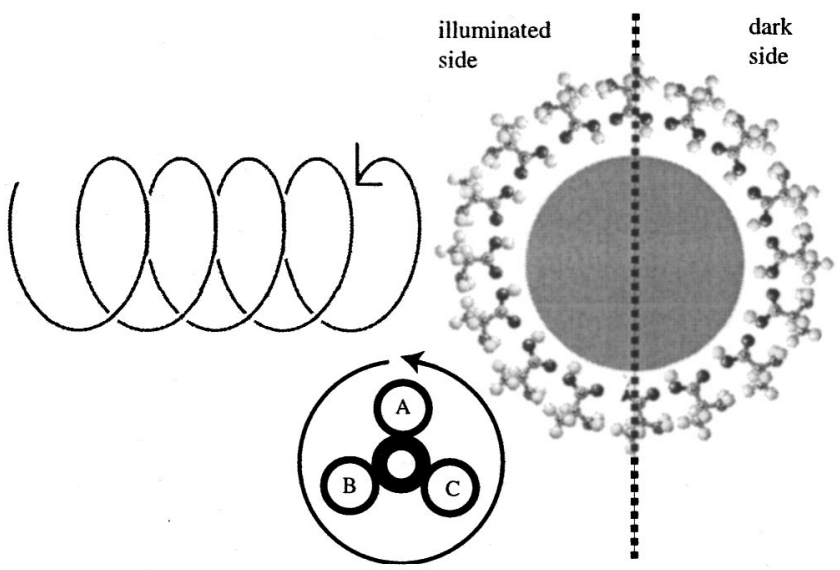

FIG. 8. A simple model for $\mathrm{CD}$ of amino acids attached to dust particles: If the molecules are attached with the same chemical group to a surface, e.g., the surface of dust particles, the molecules on the illuminated side have a preferred orientation with respect to the light. The electrical vector of the light counts the different chemical groups in the order $\mathrm{ABCABCABC}$ for the $\mathrm{L}$ form and in the order ACBACBACB for the $\mathrm{D}$ form. Thus $\mathrm{CD}$ is also possible in the dipole approximation.

tions of differently oriented molecules average out.

The position resolution of our detector allows the reconstruction of the initial momentum of the ionic fragment [19-21]. In this way, the orientation of the "fixed-in-space molecule" can be determined. While this method is not exact in the case of large molecules, it still allows one to select molecules with a preferential orientation of a particular chemical group. We call this selection partially oriented molecules.

By choosing fragments with 45 amu (or any other chemical group characterized by a specific mass) in the forward direction, the situation of amino acid molecules adsorbed on the surface of dust particles is simulated (Figs. 8 and 9). In this case, the illuminated part of the surface holds molecules which point in the propagation direction of the light and

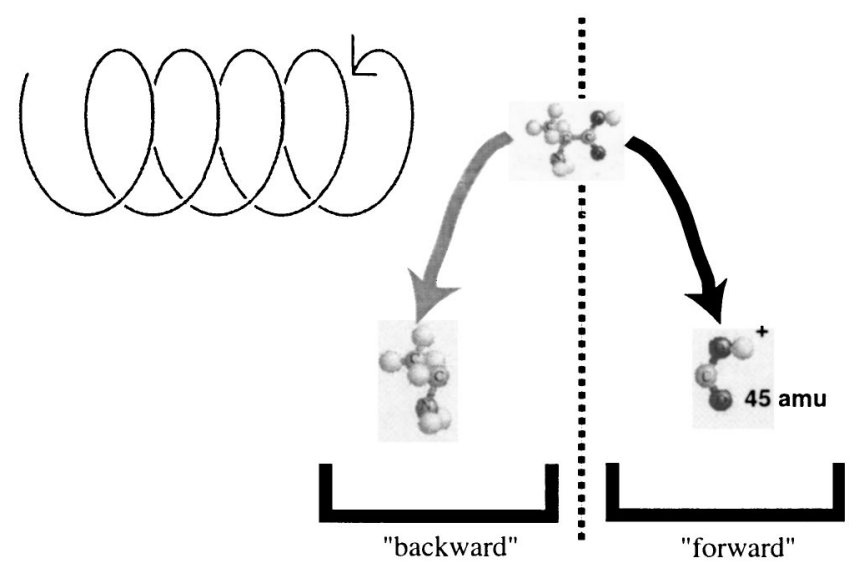

FIG. 9. With a position- and time-resolving ion detector, it is possible to select those events where a specific fragment of a specific mass is emitted in the forward direction. This incomplete measurement of the molecular orientation resembles the preferred orientation of molecules attached to spherical particles. 
which are all attached to the surface with that particular chemical group. In this situation, a CD effect is allowed even in the dipole approximation. In the plane perpendicular to the propagation direction of the light, the electrical vector will point in the direction of the different chemical groups in the order $\mathrm{ABCABCABC}$ and not $\mathrm{ACBACBACB}$ because the molecules oriented in the latter way do not lie on the illuminated side of the particle. Unlike in a real adsorbate, we can experimentally choose the chemical group that "points to the surface" arbitrarily by choosing the respective fragment masses.

We did not find any evidence for stereoselective effects in our measurements for any ionic mass. The limited statistics of the fragments being emitted in the forward direction only allow one to give an upper limit for the $\mathrm{CD}$ of the partially oriented chiral molecules of $2 \%$.

\section{SUMMARY}

We have shown that the combination of dedicated light sources and the use of a pulsed field ion time-of-flight spectrometer provides an excellent tool for performing high precision CD measurements in the gas phase. Despite this high accuracy, we found no evidence for a CD effect in the photofragmentation of the two chiral amino acids alanine and serine in the vuv region or at the $\mathrm{C}(1 s)$ edge. Our measurements set an upper limit of $0.1 \%$ to the CD asymmetry at the photon energies investigated. Theoretical predictions [24] suggest that these values are typical for the whole energy region near the $\mathrm{C}(1 s)$ edge. Tanaka and co-workers recently found evidence of $\mathrm{CD}$ values different from zero near the $\mathrm{O}(1 s)$ edge of thin L- and D-serine films [26]. The CD reaches values of up to $0.2 \%$ at some photon energies. However, these values are far too small to lead to significant enantiomeric enrichment. For partially oriented molecules, our experiment yields an upper limit for the CD of $2 \%$. Such a small CD cannot cause sizeable stereoselective effects.
TABLE I. Summary of the performed measurements and their results.

\begin{tabular}{cccc}
\hline \hline$h \nu(\mathrm{eV})$ & Amino acid & Measurement & Upper limit CD \\
\hline $10-30$ & alanine & total yield & $1 \times 10^{-3}$ \\
287 & alanine & total yield & $1 \times 10^{-3}$ \\
287 & alanine & partial total yield & $5 \times 10^{-3}$ \\
306 & serine & total yield & $1.5 \times 10^{-3}$ \\
306 & serine & partial total yield & $5 \times 10^{-3}$ \\
287 & alanine & partially oriented & $2 \times 10^{-2}$ \\
306 & serine & partially oriented & $2 \times 10^{-2}$ \\
\hline \hline
\end{tabular}

Therefore we conclude that stereoselectivity of photofragmentation of free chiral amino acids cannot account for an L excess of $10 \%$ as found in the Murchison meteorite. Strong amplification of the stereoselectivity by carrying the process to an almost complete decomposition of the original material can be ruled out in this case. In order to create a $10 \% \mathrm{~L}$ excess in the sample with a selectivity of only $0.1 \%$, the reaction would leave less than $10^{-43}$ of the original mass.

However, studies on nanometer sized amino acid aerosol particles show asymmetry values as high as $10 \%$ at a photon energy of $6.43 \mathrm{eV}[28,27]$. Even though these CD effects depend very critically on the preparation of the samples, e.g., the particle size, these and similar systems are a much more probable source for the enantiomeric excess found in the Murchison meteorite. Furthermore, Ehrenfreund and coworkers have shown that the lifetime of free amino acid molecules in the interstellar medium is comparatively short [29], supporting the thesis that the stereoselective process in the gas phase can be neglected. (See Table I.)

\section{ACKNOWLEDGMENTS}

We wish to acknowledge the support of the Deutsche Forschungsgemeinschafft and by the European Union for beamtime at BESSY II and ELETTRA.
[1] L. Pasteur, Ann. Chim. Phys. III 24, 442 (1848).

[2] B. Norden, Nature (London) 266, 567 (1977).

[3] J. Bailey et al., Science 281, 672 (1998).

[4] M. H. Engel and B. Nagy, Nature (London) 296, 837 (1982).

[5] M. H. Engel and S. A. Macko, Nature (London) 389, 265 (1997).

[6] J. R. Cronin and S. Pizzarello, Science 275, 951 (1997).

[7] W. Kuhn and E. Knopf, Z. Phys. Chem. Abt. B 7, 292 (1930).

[8] G. Balovoine, A. Moradpour, and H. B. Kagan, J. Am. Chem. Soc. 96, 5152 (1974).

[9] N. Böwering, T. Lischke, B. Schmidtke, N. Müller, T. Khalil, and U. Heinzman, Phys. Rev. Lett. 86, 1187 (2001).

[10] G. A. Garcia, L. Nahon, M. Lebech, J.-C. Houver, D. Dowek, and I. Powis, J. Chem. Phys. 119, 8781 (2003).

[11] I. Powis, J. Chem. Phys. 112, 301 (2000).

[12] I. Powis, E. E. Rennie, U. Hergenhahn, O. Kugeler, and R. Bussy-Socrate, J. Phys. Chem. A 107, 25 (2003).

[13] D. Holterkamp, W. Lange, M. Jirikowsky, and A. Benning- hoven, Appl. Surf. Sci. 17, 296 (1984).

[14] H. M. Fales, G. W. A. Milne, H. U. Winkler, H. D. Beckey, J. N. Damico, and R. Barron, Anal. Chem. 47, 207 (1975).

[15] NIST Chemistry Web-Book, edited by P. J. Linstrom and W. G. Mallard, NIST Standard Reference Database Number 69 (National Institute of Standards and Technology, Gaithersburg, MD, 2003), http://webbook.nist.gov/chemistry/(2003).

[16] A. Derossi, F. Lama, M. Piancentini, T. Prosperi, and N. Zema, Rev. Sci. Instrum. 66, 1718 (1995).

[17] M. R. Weiss, R. Follath, K. J. S. Sawhney, F. Senf, J. Bahrdt, W. Frentrup, A. Gaupp, S. Sasaki, M. Scheer, H.-C. Mertins, D. Abramsohn, F. Schäfers, W. Kuch, and W. Mahler, Nucl. Instrum. Methods Phys. Res. A 467, 449 (2001).

[18] W. C. Wiley and I. H. McLaren, Rev. Sci. Instrum. 26, 1150 (1955).

[19] N. Saito, F. Heiser, O. Hemmers, K. Wieliczek, J. Viefhaus, and U. Becker, Phys. Rev. A 54, 2004 (1996).

[20] O. Geßner, F. Heiser, N. A. Cherepkov, B. Zimmermann, and 
U. Becker, J. Electron Spectrosc. Relat. Phenom. 101-103, 113 (1999).

[21] U. Becker, O. Geßner, and A. Rüdel, J. Electron Spectrosc. Relat. Phenom. 108, 189 (2000).

[22] R. A. Mackie, R. Browning, C. J. Latimer, S. W. J. Scully, and K. F. Dunn, Chirality 14, 478 (2002).

[23] C. W. Johnson Jr., Annu. Rev. Phys. Chem. 29, 93 (1978).

[24] L. Yang, O. Plashkevych, O. Vahtras, V. Carravetta, and H. Ågren, J. Synchrotron Radiat. 6, 708 (1999).

[25] L. Alagna et al., Phys. Rev. Lett. 80, 4799 (1998).
[26] M. Tanaka et al., First Observation of Natural Circular Dichroism Spectra in VUV-Soft X-Ray Region Using Synchrotron Radiation, Symposium: Molecular Chirality 2003, Shizuoka, Japan (unpublished).

[27] J. Paul and K. Siegmann, Chem. Phys. Lett. 304, 23 (1999).

[28] J. Paul, A. Dörzbach, and K. Siegmann, Phys. Rev. Lett. 79, 2947 (1997).

[29] P. Ehrenfreund, M. P. Bernstein, J. P. Dworkin, S. A. Sandford, and L. J. Allamandola, Astrophys. J. 550, L95 (2001). 